\title{
Bypassing Both Surface Attachment and Surface Recognition Requirements for Appressorium Formation by Overactive Ras Signaling in Magnaporthe oryzae
}

\author{
Xiaoying Zhou, ${ }^{1}$ Xinhua Zhao, ${ }^{1}$ Chaoyang Xue, ${ }^{1}$ Yafeng Dai, ${ }^{2}$ and Jin-Rong $\mathbf{X u}{ }^{1}$ \\ ${ }^{1}$ Department of Botany and Plant Pathology, Purdue University, West Lafayette, IN 47907, U.S.A.; ${ }^{2}$ State Key Laboratory \\ of Crop Stress Biology for Arid Areas, Northwestern A\&F University, Yangling, Shaanxi 712100, China
}

Submitted 20 February 2014. Accepted 7 May 2014.

\begin{abstract}
Magnaporthe oryzae forms a highly specialized infection structure called an appressorium for plant penetration. In $M$. oryzae and many other plant-pathogenic fungi, surface attachment and surface recognition are two essential requirements for appressorium formation. Development of appressoria in the air has not been reported. In this study, we found that expression of a dominant active $M o R A S 2^{\mathrm{G} 18 \mathrm{~V}}$ allele in $M$. oryzae resulted in the formation of morphologically abnormal appressoria on nonconducive surfaces, in liquid suspensions, and on aerial hyphae without attachment to hard surfaces. Both the Pmk1 mitogen-activated protein kinase cascade and CAMP signaling pathways that regulate surface recognition and appressorium morphogenesis in $M$. oryzae were overactivated in the $M o R A S 2^{\mathrm{G} 18 \mathrm{~V}}$ transformant. In mutants deleted of PMK1 or CPKA, expression of $M o R A S 2^{\mathrm{G} 18 \mathrm{~V}}$ had no significant effects on appressorium morphogenesis. Furthermore, expression of dominant MoRAS2 in Colletotrichum graminicola and $\boldsymbol{C}$. gloeosporioides also caused the formation of appressoriumlike structures in aerial hyphae. Overall, our data indicate that MoRas2 functions upstream from both the cAMPPKA and Pmk1 pathways and overactive Ras signaling leads to improper activation of these two pathways and appressorium formation without surface attachment in appressorium-forming pathogens.
\end{abstract}

In many plant-pathogenic fungi that form a highly specialized infection structure called an appressorium for plant penetration, surface attachment and surface recognition are two essential requirements for appressorium development. In the rice blast fungus Magnaporthe oryzae, a model for studying fungus-plant interactions, several signal transduction pathways have been shown to be involved in the regulation of

Corresponding author: J.-R. Xu; Telephone: +1.765.496.6918; Fax: +1.765.496.6918; E-mail: jinrong@purdue.edu

$\mathrm{X}$. Zhou and X. Zhao contributed equally to this work.

The GenBank accession numbers for the MoRAS1 and MoRAS2 genes are EHA52438 and XM_00371985, respectively.

* The $e$-Xtra logo stands for "electronic extra" and indicates that three supplementary figures and one supplementary table are published online and that Figures 1, 4, 7, and 8 appear in color online.

(C) 2014 The American Phytopathological Society appressorium formation and penetration (Ebbole 2007; Wilson and Talbot 2009). Whereas cAMP signaling is important for initial surface recognition and appressorium turgor generation, the $P M K 1$ mitogen-activated protein kinase (MAPK) regulates late stages of appressorium development, penetration, and invasive growth. The cAMP-PKA pathway also is important for the generation of appressorium turgor pressure that is important for plant penetration ( $\mathrm{Li}$ et al. 2012; McDonough and Rodriguez 2012; Wilson and Talbot 2009).

Although components of the MST11-MST7-PMK1 MAPK cascade is well conserved between $M$. oryzae and the budding yeast Saccharomyces cerevisiae (Li et al. 2012), they regulate different processes in these two fungi and differ in upstream regulators and downstream targets. Unlike the yeast pheromone response pathway, two PAK kinases and $\mathrm{MgCdc} 42$ are not involved in the activation of the Mst11-Mst7-Pmk1 cascade in $M$. oryzae ( $\mathrm{Li}$ et al. 2004; Park et al. 2006). Therefore, the activation of MST11 mitogen-activated protein/extracellular signalregulated kinase remains unclear. However, it has been reported that Mst50 directly interacted with both Mst11 and Mst7, and therefore functions as an adaptor protein for the Pmk1 cascade (Park et al. 2006). Similar to the pmkl deletion mutant, the mst50 mutant was defective in appressorium formation and plant infection. Interestingly, both Mst50 and Mst11 interact with MoRas1 and MoRas2, two Ras proteins in M. oryzae, by yeast two-hybrid assays (Park et al. 2006), suggesting a role of Ras signaling in the activation of the $P M K 1$ pathway.

Ras proteins are low molecular weight GTP-binding proteins that switch between the active GTP and inactive GDP-bound statuses. They regulate cellular responses to external stimuli by activating various downstream signaling pathways. In S. cerevisiae, the two Ras proteins Ras1 and Ras2 both control the activities of adenylate cyclase (Tamanoi 2011). Intracellular acidification or exogenous glucose increases the Ras-GTP level which, in turn, activates the downstream cAMP-PKA pathway. In addition, Ras2 functions upstream from both cAMP signaling and Kss1 MAPK pathways to regulate pseudohyphal growth (Tamanoi 2011). Whereas the Ras2Val19 strain carrying the G19V mutation was stimulated in the Cdc42-Kss1dependent filamentous growth pathway (Mosch et al. 1996), expression of the RAS2Val19 allele in the gpa2 deletion mutant suppressed its defects in pseudohyphal growth (Lorenz and Heitman 1997). In the fission yeast Schizosaccharomyces pombe, a single Ras gene regulates the downstream Byr2 MAPK pathway for mating responses and the Scd1 morphogenetic pathway (Onken et al. 2006). Ras proteins also have been 
shown to play various roles in fungal development and pathogenesis processes in different fungi. In all the fungal pathogens that have been studied, Ras signaling is either essential or important for virulence (Bluhm et al. 2007; Fortwendel et al. 2004; Muller et al. 2003; Waugh et al. 2002).

In this study, we functionally characterized the two Ras genes that interacted with both Mst50 and Mst11 (Park et al. 2006). Whereas the Morasl deletion mutant had no obvious defects other than minor reduction in conidiation, MoRAS2 appeared to be an essential gene. In transformants expressing the dominant active MoRAS2 $2^{\mathrm{G} 18 \mathrm{~V}}$ allele, conidiation was significantly reduced. The MoRAS2 $2^{\mathrm{G} 18 \mathrm{~V}}$ transformant had increased Pmk1 phosphorylation and an elevated intracellular cAMP level in aerial hyphae. It formed morphologically abnormal appressoria on both hydrophilic and hydrophobic surfaces and appressorium-like structures in conidium suspensions and on aerial hyphae. Interestingly, expression of $M o R A S 2^{\mathrm{G} 18 \mathrm{~V}}$ in $\mathrm{Col}$ letotrichum graminicola and C. gloeosporioides also resulted in the formation of appressorium-like structures in aerial hyphae. These observations indicate that expression of dominant active MoRas2 may overstimulate both the cAMP signaling and Pmk1 MAPK pathways and bypass the requirements for both surface attachment and surface recognition for appressorium morphogenesis in $M$. oryzae and other appressoriumforming fungi.

\section{RESULTS}

$R A S 2$ is an essential gene in M. oryzae.

Similar to their orthologs in other fungi, MoRAS1 (MGG_09499.6) and MoRAS2 (MGG_06154.6) have five fingerprint sequence motifs (G1 to G5) for GTP/GDP-binding and a C-terminal CAAX motif. To determine their functions in M. oryzae, we attempted to generate the Moras 1 and Moras 2 deletion mutant by gene replacement (Supplementary Fig. S1). After screening over 400 transformants from independent transformations, we failed to identify Moras 2 deletion mutants. On the other hand, two Morasl mutants generated in this study were only slightly reduced in conidiation but normal in growth, appressorium formation, and plant infection. Therefore, MoRAS2 appears to be an essential gene whereas MoRAS1 may play only a minor role in conidiation. Studies in several filamentous fungi have shown that, although they are highly similar to each other, the two Ras paralogs have distinct functions and one often plays a more critical role than the other (Fernandes et al. 2008; Fillinger et al. 2002; Fortwendel et al. 2004; Nichols et al. 2009). Deletion of the major Ras gene but not the minor one is lethal in Aspergillus nidulans, Penicillium marneffei, and C. trifolii (Boyce et al. 2005; Chen et al. 2006; Fillinger et al. 2002).

\section{Abnormal appressoria formed \\ by the MoRAS2 $2^{\mathrm{G} 18 \mathrm{~V}}$ transformants.}

The dominant active allele of MoRAS2 was constructed by introducing the G18V mutation, which is equivalent to the G19V mutation in yeast (Hlavata et al. 2003). When the $M$. oryzae genome was first released, the start codon of this gene was not annotated properly and its open reading frame (ORF) had five extra amino acid residues at the $\mathrm{N}$ terminus. Strain DA66 was one of the transformants of the wild-type strain 7015 expressing the MoRAS2 ${ }^{\mathrm{G} 18 \mathrm{~V}}$ allele (Table 1). The expression level of MoRAS2 in aerial hyphae increased approximately twofold in DA66 in comparison with 70-15 (Supplementary Fig. S2). On oatmeal agar (OTA) plates, DA66 had no obvious defects in growth rate but was reduced in the production of aerial hyphae. Colonies of DA66 were heavily melanized in the center (Fig. 1A) and significantly reduced in conidiation (Table 2; Fig. 1A).

On hydrophobic surfaces, the MoRAS2 ${ }^{\mathrm{G} 18 \mathrm{~V}}$ transformant DA66 was normal in conidium germination and efficiently formed melanized appressoria (Table 2). However, appressoria formed by DA66 were morphologically abnormal. Compared with normal dome-shaped appressoria, the majority of appressoria formed by DA66 had irregular shapes (Fig. 1B). Some of them appeared to be formed subapically or in chains. These observations indicated that expression of MoRAS2 ${ }^{\mathrm{G} 18 \mathrm{~V}}$ may result in improper regulation of appressorium morphogenesis.

\section{Transformant DA66 formed appressoria on hydrophilic surfaces.}

Recognition of hydrophobic surfaces is an important signal for appressorium formation in $M$. oryzae. On the hydrophilic surface of GelBond membranes, germ tubes of 70-15 rarely formed appressoria after incubation for $24 \mathrm{~h}$. Under the same conditions, over $90 \%$ of the DA66 germ tubes formed appressoria (Table 2; Fig. 1C). Because DA66 formed appressoria efficiently on both hydrophobic and hydrophilic surfaces, expression of the MoRAS2 ${ }^{\mathrm{G} 18 \mathrm{~V}}$ allele must be sufficient to bypass the requirement for attachment to hydrophobic surfaces for appressorium formation.

Table 1. Wild-type and mutant strains of Magnaporthe oryzae used in this study

\begin{tabular}{|c|c|c|}
\hline Strain & Genotype description & Reference \\
\hline $70-15$ & Wild-type, MAT1-1 & Chao and Ellingboe 1991 \\
\hline Guy11 & Wild-type, MAT1-2 & Leung et al. 1988 \\
\hline Ku80 & $\Delta M g k u 80$ mutant of Guy 11 & Villalba et al. 2008 \\
\hline nn78 & $\Delta p m k 1$ mutant of Guy11 & Xu and Hamer 1996 \\
\hline I-27 & $\Delta c p k A$ mutant of $70-15$ & Mitchell and Dean 1995 \\
\hline DA5 & $M S T 7^{\mathrm{S} 212 \mathrm{D} T 216 \mathrm{E}}$ transformant of $70-15$ & Zhao et al. 2005 \\
\hline DA66 & MoRAS2 $2^{\mathrm{G} 18 \mathrm{~V}}$ transformant of $70-15$ & This study \\
\hline HZ90 & MoRAS2 $2^{\mathrm{G} 18 \mathrm{~V}}$ transformant of $70-15$ & This study \\
\hline HZ91 & MoRAS2 ${ }^{\mathrm{G} 18 \mathrm{~V}}$ transformant of $70-15$ & This study \\
\hline HZ92 & MoRAS2 $2^{\mathrm{G} 18 \mathrm{~V}}$ transformant of $70-15$ & This study \\
\hline CX77 & $\Delta r a s 1$ mutant of $70-15$ & This study \\
\hline DAG-1 & Transformant of DA66 expressing GASI-GFP & This study \\
\hline DAG-6 & Transformant of DA66 expressing GAS1-GFP & This study \\
\hline DA1-3 & MoRAS2 ${ }^{\mathrm{G} 18 \mathrm{~V}}$ transformant of Colletotrichum graminicola & This study \\
\hline DA2-2 & MoRAS2 $2^{\mathrm{G} 18 \mathrm{~V}}$ transformant of C. gloeosporioides & This study \\
\hline DA4-1 & MoRAS2 $2^{\mathrm{G} 18 \mathrm{v}}$ transformant of Fusarium graminearum & This study \\
\hline DA-P1 & MoRAS2 $2^{\mathrm{G} 18 \mathrm{~V}}$ transformant of the pmk1 mutant nn78 & This study \\
\hline DA-P2 & $M o R A S 2^{\mathrm{G} 18 \mathrm{~V}}$ transformant of the $p m k 1$ mutant $\mathrm{nn} 78$ & This study \\
\hline $\mathrm{DA}-\mathrm{C} 1$ & MoRAS2 $2^{\mathrm{G} 18 \mathrm{~V}}$ transformant of the $c p k A$ mutant I-27 & This study \\
\hline $\mathrm{DA}-\mathrm{C} 2$ & $M o R A S 2^{\mathrm{G} 18 \mathrm{~V}}$ transformant of the $c p k A$ mutant I-27 & This study \\
\hline
\end{tabular}




\section{Expression of MoRAS2 ${ }^{\mathrm{G} 18 \mathrm{~V}}$ bypasses} surface attachment for appressorium formation.

In $M$. oryzae, it has been reported that attachment to hard surfaces in the initial $2 \mathrm{~h}$ is required to form appressoria (Liu et al. 2007). To test whether transformant DA66 could form appressoria without surface attachment, conidia of both DA66 and 70-15 were incubated in hanging drops to avoid direct contact with hard surfaces (Fig. 2A). After incubation for $24 \mathrm{~h}$,
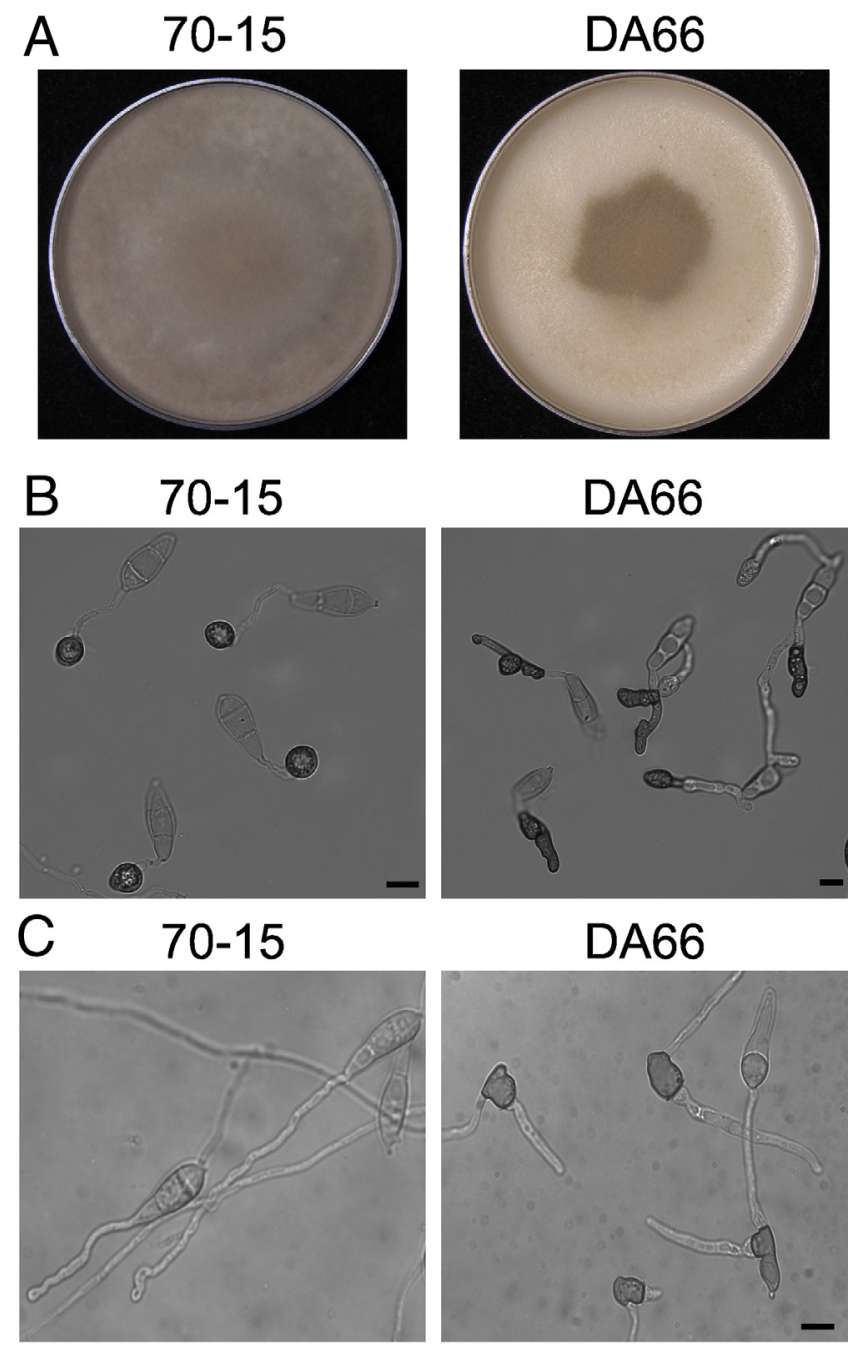

Fig. 1. Abnormal colony and appressorium morphology of the MoRAS2 $2^{\mathrm{G} 18 \mathrm{~V}}$ transformant DA66. A, One-week-old oatmeal agar cultures of the wildtype strain 70-15 and DA66. Enhanced melanization was observed in the center of DA66 colonies (arrow). B, In comparison with 70-15, DA66 formed morphologically abnormal appressoria on hydrophobic surfaces. C, On the hydrophilic surface of GelBond membranes, 70-15 failed to form appressoria. Bar $=10 \mu \mathrm{m}$. conidia of 70-15 aggregated at the bottom of conidium drops and produced long germ tubes without tip differentiation (Fig. 2B). Under the same conditions, DA66 conidia germinated and formed morphologically abnormal appressoria (Fig. 2B). These results indicated that dominant active MoRAS2 bypassed the surface attachment requirement for appressorium formation.

The same hanging drop method was used to assay for appressorium formation with conidia of transformant DA5 that expresses the dominant active $M S T 7^{\mathrm{S} 212 \mathrm{D}}{ }^{\mathrm{T} 216 \mathrm{E}}$ allele (Zhao et al. 2005). Although transformant DA5 formed appressoria on both hydrophobic and hydrophilic surfaces (Zhao et al. 2005), no appressoria were observed in the conidium hang-
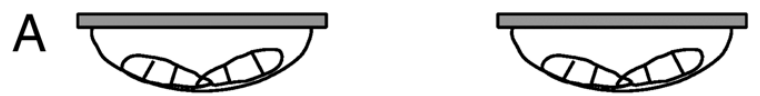

B

\section{$70-15$}
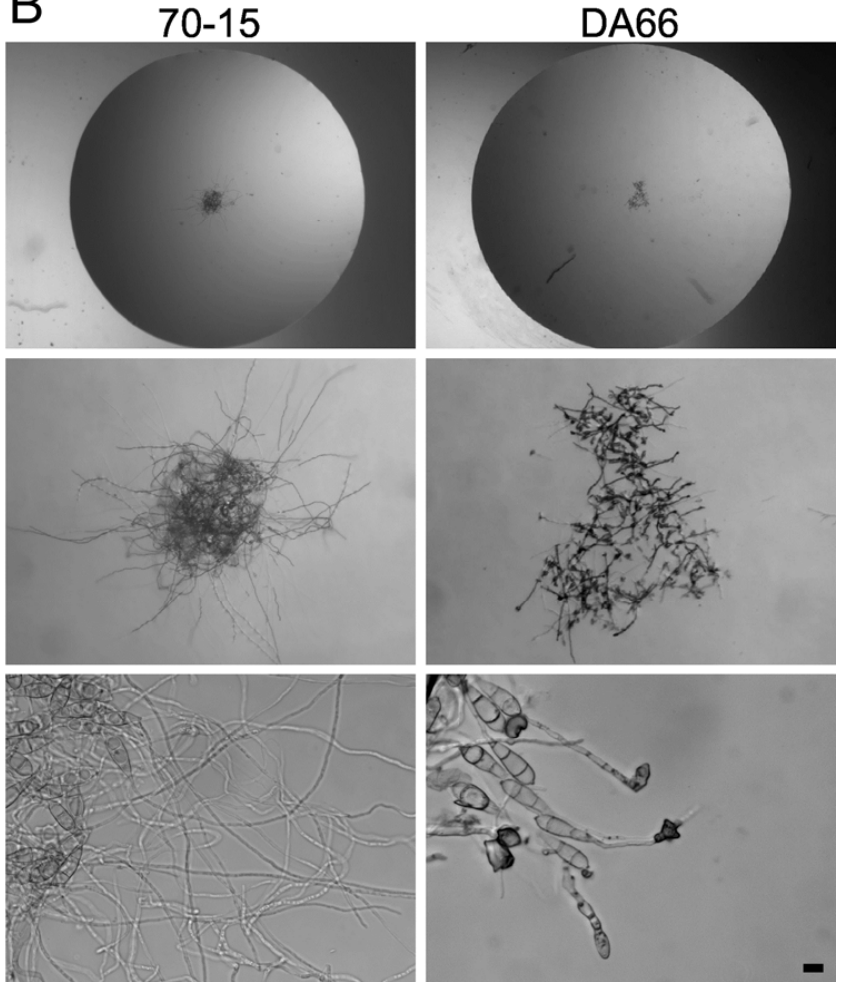

Fig. 2. Appressorium formation assays with hanging drops of conidium suspensions. A, Drops $(20 \mu \mathrm{l})$ of conidium suspensions $\left(2 \times 10^{4}\right.$ spores $/ \mathrm{ml}$ ) were placed over concave slide glasses and incubated upside down in a moisture chamber. B, After incubation for $36 \mathrm{~h}$, conidium suspensions were examined under subjective lens of different magnifications. Conidia of 70-15 produced long germ tubes. Germ tubes of DA66 tended to be short and often formed melanized appressoria of abnormal morphology at the tip. Bar $=10 \mu \mathrm{m}$.

Table 2. Growth rate, conidiation, and appressorium formation of Magnaporthe oryzae strains used in this study

\begin{tabular}{|c|c|c|c|c|}
\hline \multirow[b]{2}{*}{ Strain } & \multirow[b]{2}{*}{ Growth rate $(\mathrm{mm} / \mathrm{day})^{\mathrm{z}}$} & \multirow[b]{2}{*}{ Conidiation $\left(\times 10^{4}\right.$ conidia/plate $)$} & \multicolumn{2}{|c|}{ Appressorium formation $(\%)^{\mathrm{y}}$} \\
\hline & & & Hydrophobic & Hydrophilic \\
\hline $70-15$ & $3.1 \pm 0.2 \mathrm{~A}$ & $250.2 \pm 66.2 \mathrm{~B}$ & $93.6 \pm 6.0 \mathrm{~A}$ & $1.2 \pm 1.0$ \\
\hline DA66 & $2.9 \pm 0.3 \mathrm{~A}$ & $5.6 \pm 3.6 \mathrm{E}$ & $92.9 \pm 5.5 \mathrm{~A}$ & $90.9 \pm 7.2$ \\
\hline DA-P1 & $2.1 \pm 0.2 \mathrm{~B}$ & $240.7 \pm 93.8 \mathrm{~B}$ & 0 & 0 \\
\hline DA-C1 & $3.2 \pm 0.2 \mathrm{~A}$ & $390.3 \pm 57.8 \mathrm{~A}$ & $42.1 \pm 1.9 \mathrm{C}$ & $1.8 \pm 0.3$ \\
\hline Guy11 & $3.1 \pm 0.2 \mathrm{~A}$ & $480.4 \pm 37.8 \mathrm{~A}$ & $96.9 \pm 2.5 \mathrm{~A}$ & $1.0 \pm 0.9$ \\
\hline
\end{tabular}

${ }^{\mathrm{x}}$ Numbers followed by the same letter indicate that there was no significant difference. Different letters were used to mark statistically significant differences $(P=0.05)$.

${ }^{\mathrm{y}}$ Percentages of germ tubes formed appressoria on the hydrophobic and hydrophilic surfaces of GelBond membranes.

${ }^{\mathrm{z}}$ Average growth rate and (mean \pm standard deviation) were calculated from three independent measurements. 
ing-drop assays (Supplementary Fig. S3). These observations indicated that constitutive activation of the Pmk1 MAPK by

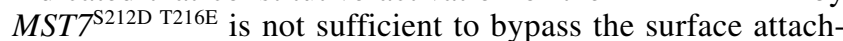
ment requirement for appressorium formation. Therefore, expression of the dominant active MoRAS2 allele must have additional effects other than overstimulation of the Pmk1 pathway in $M$. oryzae.

\section{Appressorium-like structures formed}

by the $M o R A S 2^{\mathrm{G} 18 \mathrm{~V}}$ transformant in the air.

Because of enhanced melanization, we examined aerial hyphae and conidiophores harvested from the center of 2-weekold OTA cultures of strain DA66. Interestingly, the formation of melanized, appressorium-like structures were observed in aerial hyphae (Fig. 3A). Similar to appressoria formed by DA66 in hanging drop assays, these melanized structures developed in the air had irregular morphology. Most of them were formed at the tip or subapical regions of aerial hyphae or conidiophores (Fig. 3A). A septum was formed to delimit these irregular melanized structures, which is similar to appressorium differentiation by germ tubes (Saunders et al. 2010). Cryo-scanning electron microscopy (SEM) examination confirmed the formation of appressorium-like structures by transformant DA66 in the air (Fig. 3B)

\section{GAS1 is expressed in appressorium-like structures formed in the air.}

To further confirm that melanized hyphal tips were appressorium-like structures, a GAS1-green fluorescent protein (GFP) reporter construct generated in a previous study (Xue et al. 2002) was transformed into strain DA66. Although GAS1 transcripts were present in other stages (Soanes et al. 2012), GFP signals were increased and became visible in the cytoplasm in late stages of appressoria in the GAS1-GFP trans-
A
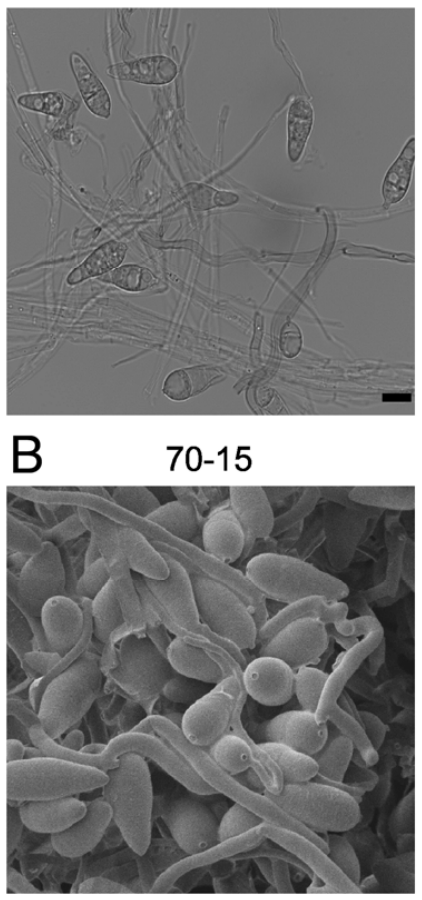

\section{DA66}

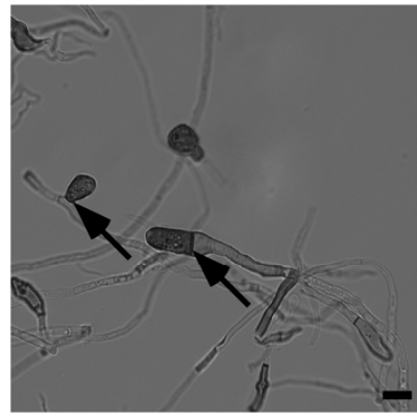

DA66

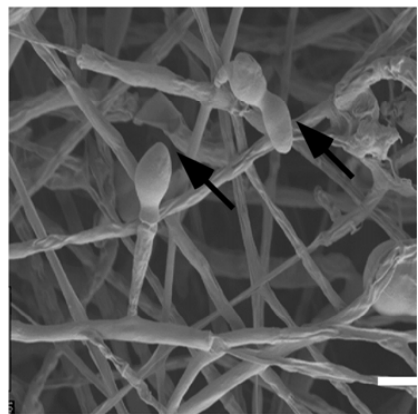

Fig. 3. Aerial hyphae and conidiophores were harvested from the center of 1-week-old oatmeal agar cultures and examined by $\mathbf{A}$, differential interference contrast microscopy and $\mathbf{B}$, cryo-scanning electron microscopy. Bar = $10 \mu \mathrm{m}$. Whereas abundant conidia were formed by $70-15$, transformant DA66 rarely produced conidia but developed appressorium-like structures (arrows) at the tip of aerial hyphae and conidiophores. formants (Xue et al. 2002). Two zeocin-resistant transformants of DA66 carrying a single copy of the GAS1-GFP construct (DAG-1 and DAG-6) were identified. Approximately $31.2 \pm$ $5.6 \%$ of appressorium-like structures formed in the air by these two transformants had GFP signals in the cytoplasm (Fig. 4A), which is similar to the expression of GAS1-GFP in appressoria formed by the wild type (Xue et al. 2002). When aerial hyphae were fixed and examined by transmission electron microscopy (TEM), appressorium-like structures formed by DA66, similar to appressoria formed by germ tubes on hydrophobic surfaces, had the melanin layer (Fig. 4B). These data further indicate that these appressorium-like structures formed in the air have basic characteristics of appressoria.

\section{MoRas2 functions upstream}

from the Pmk1 MAPK pathway.

Among the three M. oryzae genes (MST50, MST11, and $M A C 1$ ) with the Ras association (RA) domain, two are compo-
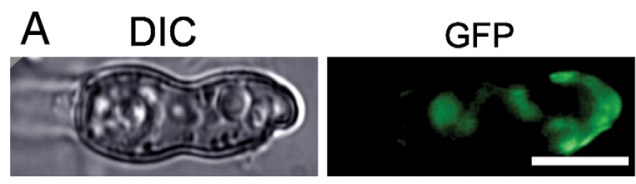

B
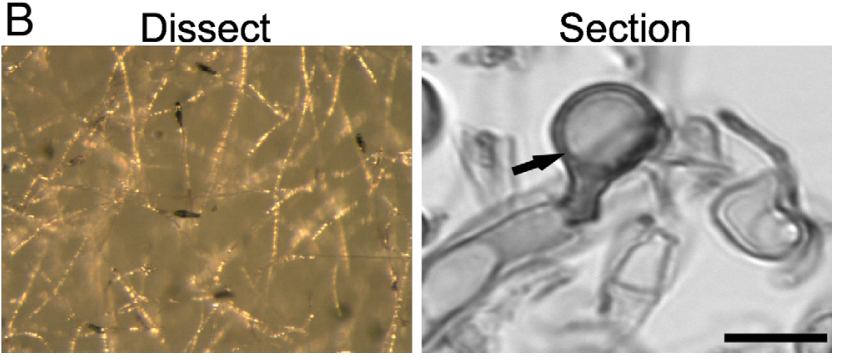

Fig. 4. Expression of GAS1-green fluorescent protein (GFP) and formation of a melanin layer in melanized hyphal compartments. A, Appressoriumlike structures formed in aerial hyphae of DAG-1, a transformant of DA66 expressing the GAS1-GFP construct, were examined by differential interference contrast and epifluorescence microscopy. B, Aerial hyphae of DA66 were examined with a dissecting microscope and by transmission electron microscopy. Arrows pointed to melanized appressorium-like structures and the melanin layer. Bar $=10 \mu \mathrm{m}$.

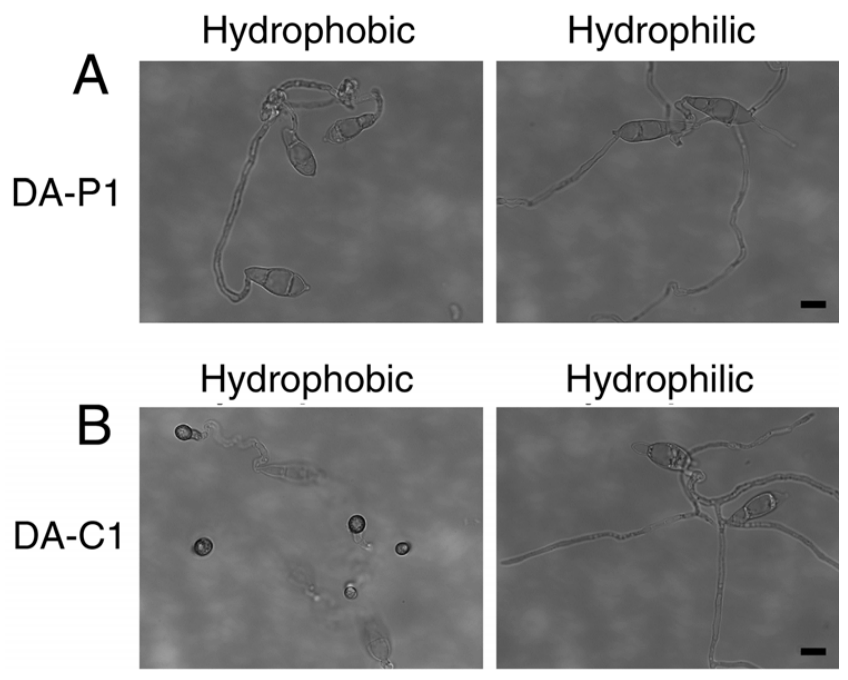

Fig. 5. Appressorium formation assays with transformants of the $\Delta p m k 1$ and $\triangle c p k A$ mutants expressing the $M o R A S 2^{\mathrm{G} 18 \mathrm{~V}}$ allele. A, The $\Delta p m k 1 /$ $M o R A S 2^{\mathrm{G} 18 \mathrm{~V}}$ transformant failed to form appressoria on hydrophobic or hydrophilic surfaces. B, The $\triangle c p k A / M o R A S 2^{\mathrm{G} 18 \mathrm{~V}}$ transformant formed appressoria on hydrophobic but not hydrophilic surfaces. 
nents of the Pmk1 MAPK pathway (Park et al. 2006; Zhao et al. 2005). To determine the functional relationship between MoRas2 and Pmk1, we transformed the MoRAS2 ${ }^{\mathrm{G} 18 \mathrm{~V}}$ allele into the pmk1 mutant nn78 (Xu and Hamer 1996). The resulting pmk1/MoRAS2 $2^{\mathrm{G} 18 \mathrm{~V}}$ transformants DA-P1 and DA-P2 (Table 1), like the pmk1 mutant, failed to form appressoria on hydrophobic or hydrophilic surfaces (Fig. 5A; Table 2), indicating that Pmk1 is required for improper appressorium morphogenesis in the MoRAS2 $2^{\mathrm{G} 18 \mathrm{~V}}$ transformant.

To further investigate the role of MoRas2 in appressorium development, we assayed the activation of Pmk1 with an antiTpEY antibody. In proteins isolated from vegetative hyphae harvested from 2-day-old liquid complete medium (CM) cultures, no significant difference in the phosphorylation of Pmk1 was observed between the wild type strain 70-15 and transformant DA66 (Fig. 6A). However, in proteins isolated from aerial hyphae harvested from 2-week-old OTA cultures, the phosphorylation level of Pmk1was significantly higher in DA66 than in 70-15 (Fig. 6A), which is consistent with the observa-
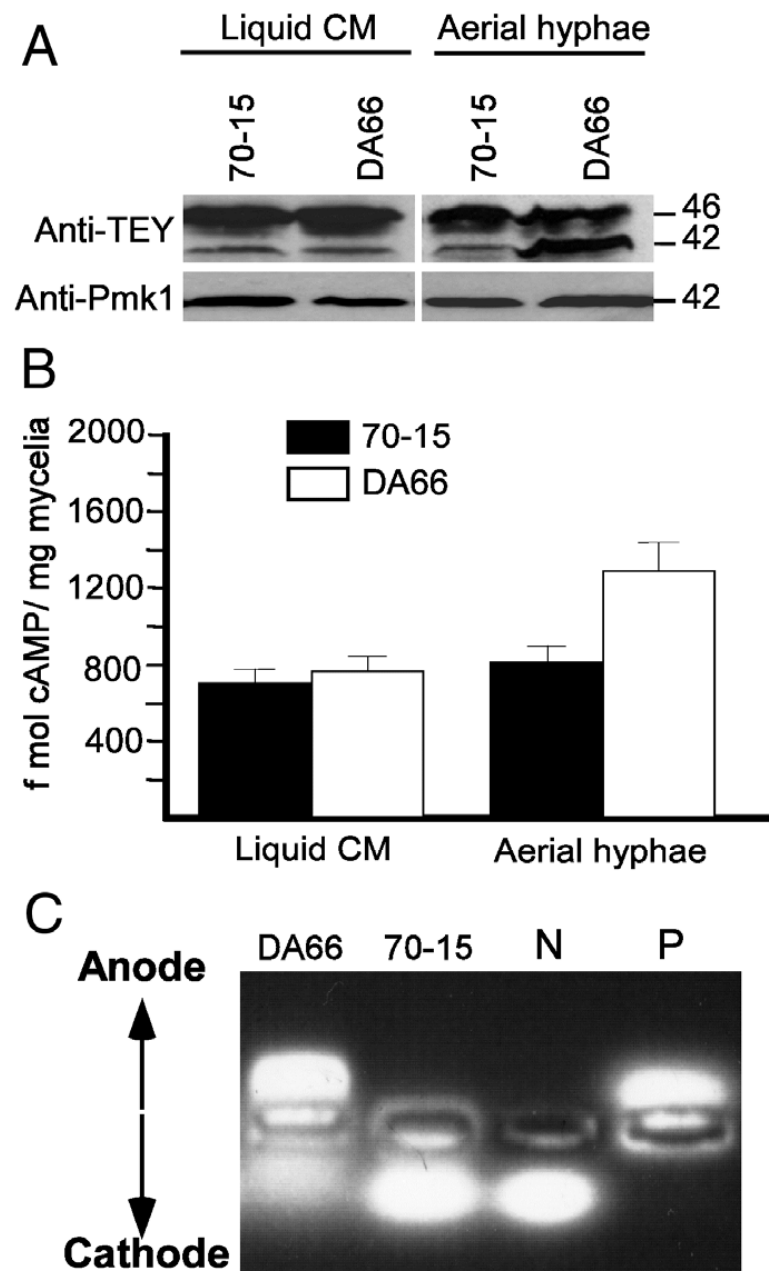

Fig. 6. Assays for Pmk1 activation and intracellular cAMP. A, Western blot analysis for the expression and activation of Pmk1. A similar band was detected by an anti-Pmk1 antibody in proteins isolated from aerial hyphae and liquid CM cultures of 70-15 and DA66. When detected with an anti-TpEY antibody, Pmk1 but not Mps1 phosphorylation was increased in proteins isolated from aerial hyphae of DA66. B, An elevated intracellular cAMP level was detected in aerial hyphae but not liquid CM cultures of DA66. C, Assays for PKA activities in proteins isolated from aerial hyphae with the PepTag A1 PKA substrate peptide. On a $0.8 \%$ agarose gel, phosphorylated peptides in DA66 samples migrated toward the anode $(+)$. In 70-15 samples, unphosphorylated peptides migrated toward the cathode $(-)$. $\mathrm{N}=$ nonphosphorylated sample control and $\mathrm{P}=$ phosphorylated sample control. tion that DA66 but not 70-15 formed appressorium-like structures in the air. In the same protein samples, the phosphorylation level of the Mps1 MAPK was similar between 70-15 and DA66, indicating that MoRAS2 $2^{\mathrm{G} 18 \mathrm{~V}}$ specifically overstimulated the Pmk1 but not Mps1 MAPK pathway.

\section{MoRas2 is also involved in cAMP signaling.}

In $M$. oryzae, the Mac1 adenylate cyclase (Choi and Dean 1997) is one of the three proteins that have the RA domain. To determine the functional relationship between MoRas2 and cAMP signaling, we transformed the MoRAS2 ${ }^{\mathrm{G} 18 \mathrm{~V}}$ allele into the $c p k A$ mutant I-27 (Mitchell and Dean 1995). The resulting transformant, DA-C1, formed appressoria on hydrophobic but not on hydrophilic surfaces (Fig. 5B; Table 2). In addition, transformant DA-C1, similar to the cpkA mutant, tended to produce smaller appressoria. Therefore, expressing the dominant active MoRAS2 $2^{\mathrm{G} 18 \mathrm{~V}}$ allele failed to suppress the defects of the cpkA mutant in appressorium formation, indicating that $M o R A S 2$ also functions upstream from the cAMP signaling pathway.

To confirm this observation, we measured the intracellular cAMP level and PKA activities in the MoRAS2 $2^{\mathrm{G} 18 \mathrm{~V}}$ transformant. In proteins isolated from vegetative hyphae, the intracellular cAMP level was similar between transformant DA66 and 70-15 (Fig. 6B). However, in comparison with the wild type, transformant DA66 had an elevated intracellular cAMP level in aerial hyphae harvested from 2-week-old OTA cultures. In addition, PKA activities were significantly higher in transformant DA66 than in 70-15 (Fig. 6C). These results confirmed that expression of the MoRAS2 ${ }^{\mathrm{G} 18 \mathrm{~V}}$ allele stimulated the cAMP-PKA pathway in M. oryzae.

\section{Transformants expressing the $M g R A S 2^{\mathrm{G} 18 \mathrm{~V}}$ allele are nonpathogenic.}

Because the $M g R A S 2^{\mathrm{G} 18 \mathrm{~V}}$ transformant rarely produced conidia, we conducted infection assays with culture blocks. On seedlings inoculated with culture blocks of transformant DA66, no lesions were formed on the intact rice leaves (Fig. 7A). On wounded leaves, DA66 also failed to cause blast lesions outside the wounding sites (Fig. 7B). Under the same conditions, $70-15$ caused lesions on both intact and wounded leaves (Fig. 7). Therefore, although it was overstimulated in appressorium formation, the $M g R A S 2^{\mathrm{G} 18 \mathrm{~V}}$ transformant was defective in plant infection.

Expression of $M o R A S 2^{\mathrm{G} 18 \mathrm{~V}}$ also stimulates the formation of aerial appressoria in other appressorium-forming fungi.

MoRAS2 orthologs are well conserved in filamentous ascomycetes. To determine whether the regulatory function of MoRAS2 in appressorium formation is conserved in other plant-pathogenic fungi, we transformed the MoRAS2 ${ }^{\mathrm{G} 18 \mathrm{~V}}$ allele into the wild-type strains of $C$. graminicola and C. gloeosporioides. The resulting transformants of $C$. graminicola and $C$. gloeosporioides, DA1-3 and DA2-2, respectively (Table 1), contained a single copy of MoRAS2 ${ }^{\mathrm{G} 18 \mathrm{~V}}$. They had no obvious changes in growth rate or colony morphology. Similar to DA66 in $M$. oryzae, melanized appressorium-like structures were observed on the tip of aerial hyphae in the MoRAS2 $2^{\mathrm{G} 18 \mathrm{~V}}$ transformants DA1-3 and DA2-2 (Fig. 8).

We also transformed the MoRAS2 ${ }^{\mathrm{G} 18 \mathrm{~V}}$ construct into a nonappressorium-forming fungus, Fusarium graminearum. The resulting transformant DA4-2 (Table 1) was confirmed by polymerase chain reaction (PCR) to contain the MoRAS2 $2^{\mathrm{G} 18 \mathrm{~V}}$ construct. It grew slightly more slowly than the wild-type strain on V8 agar plates but failed to produce melanized appressoria in aerial hyphae, which is not surprising because $F$. graminearum doesn't form appressoria. Therefore, expression of 
$M o R A S 2^{\mathrm{G} 18 \mathrm{~V}}$ only resulted in appressorium formation on aerial hyphae in appressorium-forming fungi.

\section{DISCUSSION}

Like many other fungi, the $M$. oryzae genome contains two Ras genes. Whereas deletion of MoRAS1 had no obvious phenotypes, MoRAS2 appeared to be an essential gene and expression of a dominant active allele of MoRAS2 resulted in pleiotropic defects. Therefore, MoRAS2 must play a more critical role than MoRAS1 in growth and development in M. oryzae, which is similar to what has been reported in other filamentous fungi, including A. nidulans, Cryptococcus neoformans, and Schizophyllum commune (Fillinger et al. 2002; Schubert et al. 2006; Waugh et al. 2002). In all these fungi, one of the two paralogous Ras gene is more important or has a higher expression level than the other. In contrast, both Ras1 and Ras2 function to activate adenylate cyclase in Saccharomyces cerevisiae. Although none of them is essential, the ras 1 ras 2 double mutant is not viable (Tamanoi 2011). In Ustilago maydis, two Ras proteins have distinct functions. Whereas Ras 2 controls filamentous growth, Ras1 is involved in pheromone responses (Lee and Kronstad 2002; Muller et al. 2003).

The cAMP signaling and Pmk1 MAPK pathways are known to be involved in surface recognition and appressorium morphogenesis in M. oryzae (Ebbole 2007; Li et al. 2012). In aerial hyphae of the MoRAS2 ${ }^{\mathrm{G} 18 \mathrm{~V}}$ transformant, Pmk1 phosphorylation and intracellular cAMP levels were increased, indicating
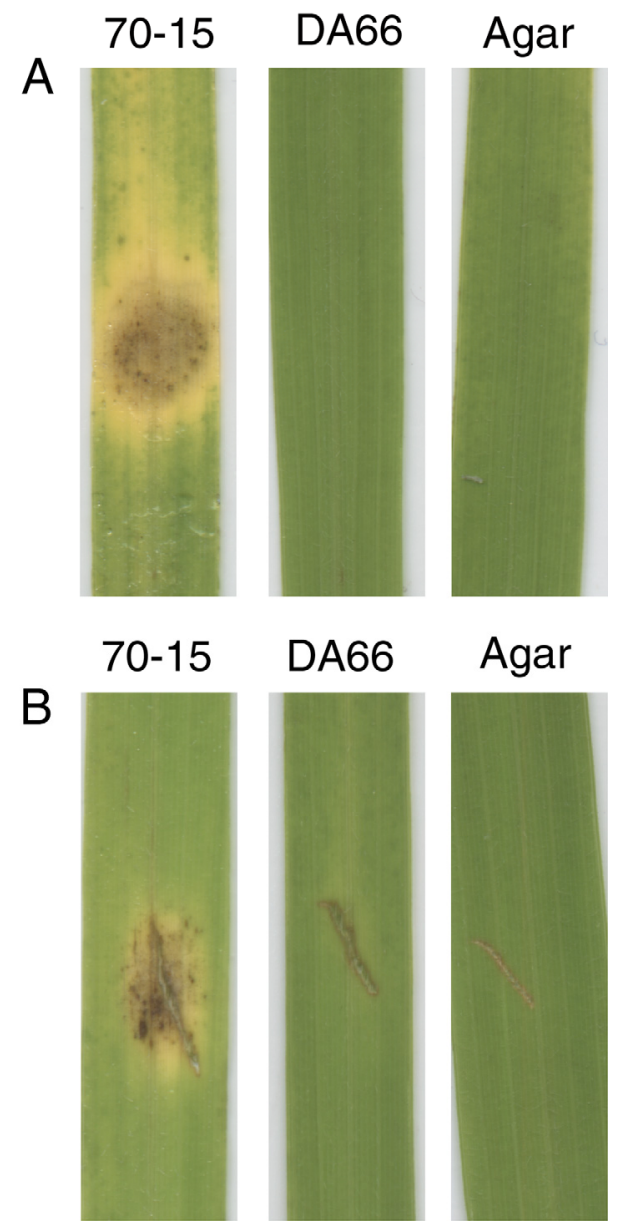

Fig. 7. Plant infection assays with the $M o R A S 2^{\mathrm{G} 18 \mathrm{~V}}$ transformant. A, Intact and $\mathbf{B}$, wounded rice leaves were inoculated with water agar (control) or culture blocks of the wild type (70-15) and MoRAS2 $2^{\mathrm{G} 18 \mathrm{~V}}$ transformant (DA66) strains. Symptoms were examined 6 days postinoculation. that expression of MoRAS2 $2^{\mathrm{G} 18 \mathrm{~V}}$ overstimulated both cAMP signaling and Pmk1 MAPK pathways, which is consistent with the formation of appressoria on hydrophilic surfaces and in the air. Therefore, MoRas2 is likely involved in both Pmk1 and cAMP signaling pathways in $M$. oryzae. In $S$. cerevisiae, expression of Ras $2^{\text {v19 }}$ increased the intracellular cAMP level and stimulated filamentous growth but had no effect on the mating pheromone pathway (Mosch et al. 1999; Tamanoi 2011). In Candida albicans, Ras1 is known to regulate both cAMP and MAPK pathways as well (Zhu et al. 2009). In Cryptococcus neoformans, Ras1 likely mediates cross-talk between the Cek1 MAPK and cAMP signaling pathways. The ras 1 deletion mutant was defective in mating, agar adherence, and virulence. Its defect in mating was fully suppressed by overexpression of GPB1 that regulates the Cpk1 MAPK pathway but only partially rescued by exogenous cAMP (Shapiro et al. 2011).

Because the phosphorylation level of Mps1 was not affected in the MoRAS2 $2^{\mathrm{G} 18 \mathrm{~V}}$ transformant, MoRas2 likely plays a specific role in the activation of the Pmk1 pathway. Interestingly, Pmk1 activation and the intracellular cAMP level were not affected in vegetative hyphae harvested from liquid CM cultures of the MoRAS2 $2^{\mathrm{G} 18 \mathrm{~V}}$ transformant. Consistent with that, we noticed that melanized appressorium-like structures were not formed at the tip of vegetative hyphae in liquid cultures. It is likely that melanized hyphal tips were related to aerial hyphal growth or conidium development, which does not occur in liquid cultures. Expression of $M o R A S 2^{\mathrm{G} 18 \mathrm{~V}}$ resulted in the formation of appressorium-like structures instead of conidia, which was consistent with the fact that the MoRAS2 $2^{\mathrm{G} 18 \mathrm{~V}}$ transformant rarely produced conidia (Fig. 3; Table 2). In Neurospora crassa, the band mutation that resulted in dominant active Ras $1^{\text {bd }}$ also affected cell differentiation and asexual development (Belden et al. 2007). In A. fumigatus, development of conidiophore and sterigmata was affected in the transformant expressing a dominant active RasA (Fortwendel et al. 2004).

Although appressorium formation was enhanced, the $M o R A S 2^{\mathrm{G} 18 \mathrm{~V}}$ transformant was nonpathogenic in infection assays with intact and wounded leaves (Fig. 7). Similar phenomenon was observed in the transformant expressing the
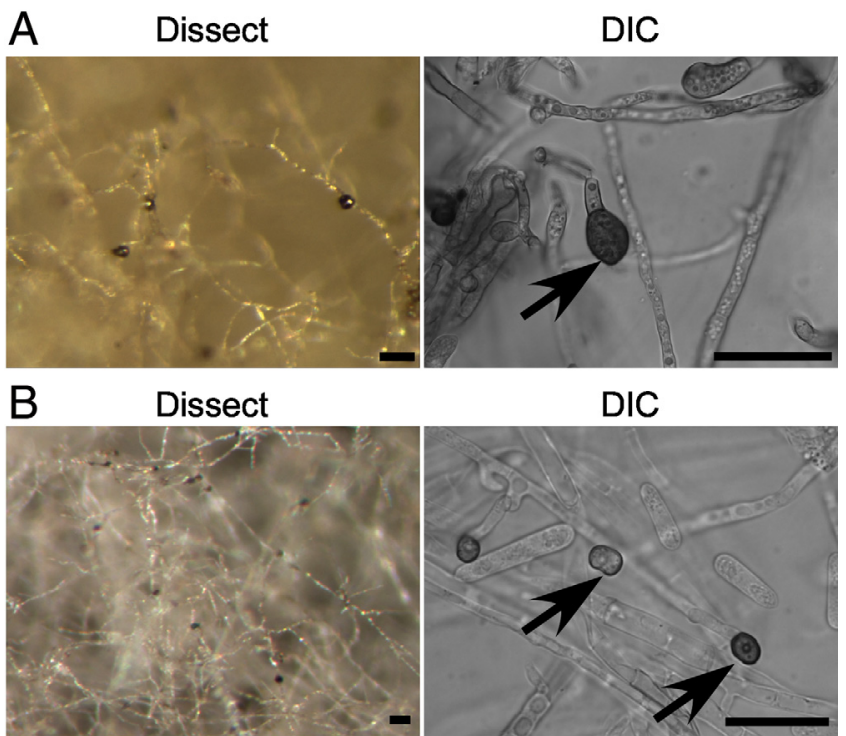

Fig. 8. Expression of the $M g R A S 2^{\mathrm{G} 18 \mathrm{~V}}$ allele in Colletotrichum graminicola and C. gloeosporioides. Aerial hyphae from 2-week-old cultures of the $M g R A S 2^{\mathrm{G} 18 \mathrm{~V}}$ transformants of A, C. graminicola and B, C. gloeosporioides were examined by dissecting (left) and differential interference contrast microscopy. Arrows point to melanized appressoria. Bar $=30 \mu \mathrm{m}$. 
$M S T 7^{\mathrm{DA}}$ allele that functions upstream from the Pmk1 MAPK (Zhao and $\mathrm{Xu}$ 2007). Because constitutive activation of the Pmk1 MAPK cascade resulted in loss of pathogenicity, it is likely that Pmk1 MAPK needs to be properly regulated during different infection stages. Appressorial penetration, development of invasive hyphae, and invasive growth may require the coordinated regulation between cAMP signaling and Pmk1 MAPK pathways in $M$. oryzae. Dominant active Ras mutations also have been shown to affect virulence in other fungal pathogens, including $C$. neoformans and $U$. maydis (Lee and Kronstad 2002; Lengeler et al. 2000; Xie et al. 2013).

One phenomenon we observed in the MoRAS2 ${ }^{\mathrm{G} 18 \mathrm{~V}}$ transformant is that it was relatively unstable during vegetative growth. The efficiency of appressorium formation on hydrophilic surfaces and the formation of melanized hyphal tips were often reduced in subcultures. In $M$. oryzae, the mutants blocked in the Pmk1 MAPK cascade, such as the pmkl, mst7, and mst 11 mutants, are stable. However, mutants defective in cAMP signaling, such as the $m g b l$, macl, and capl mutants, are known to produce spontaneous suppressors (Fang and Dean 2000; Nishimura et al. 2003; Zhou et al. 2012). Spontaneous mutations that occurred in the cAMP-PKA pathway or other downstream effectors of MoRas 2 may partially suppress the phenotypes of the MoRAS2 $2^{\mathrm{G} 18 \mathrm{~V}}$ transformant. However, post-translational modifications such as phosphorylation also are known to affect Ras activities (Tamanoi 2011), and the A59T mutation in the dominant active H-Ras attenuates the efficiency of fibroblast transformation as a suppressor (Zisoulis and Kansas 2004). Therefore, suppressors of dominant active MoRas2 in $M$. oryzae may result from postmodifications or point mutations of MoRAS2 $2^{\mathrm{G} 18 \mathrm{~V}}$

Due to the observation of the instability of the MoRAS2 $2^{\mathrm{G} 18 \mathrm{~V}}$ transformants, all the data presented in this publication were generated with subcultures from the original transformants. Although Table 1 listed only four of them, we isolated over 10 MoRAS2 $2^{\mathrm{G} 18 \mathrm{~V}}$ transformants from different transformants. All of them had similar phenotypes, suggesting that it is unlikely for the observed phenotypes to be caused by positional effects of the MoRAS2 $2^{\mathrm{G} 18 \mathrm{~V}}$ integration sites. Nevertheless, quantitative reverse-transcriptase PCR assays showed that the expression level of MoRAS2 in aerial hyphae increased approximately twofold in the MoRAS2 $2^{\mathrm{G} 18 \mathrm{~V}}$ transformant. Although the transforming MoRAS2 $2^{\mathrm{G} 18 \mathrm{~V}}$ construct was under the control of its native promoter, it is possible that some of the phenotypes observed in these transformants are related to the expression of MoRAS2.

Surface attachment and recognition are critical for appressorium development in $M$. oryzae and other fungal pathogens (Lee et al. 2003; Wilson and Talbot 2009). In this study, we found that the MoRAS2 $2^{\mathrm{G} 18 \mathrm{~V}}$ transformant efficiently formed appressoria on hydrophilic surfaces and in hanging conidium drop assays. Expression of $M o R A S 2^{\mathrm{G} 18 \mathrm{~V}}$ also resulted in the formation of appressorium-like structures on aerial hyphae (Fig. 4). These results indicated that the requirements for both surface attachment and surface recognition for appressorium formation were bypassed by dominant active MoRas2. Expression of the MoRAS2 ${ }^{\mathrm{G} 18 \mathrm{~V}}$ allele in C. graminicola and C. gloeosporioides also resulted in the formation of melanized, appressorium-like structures at the tip of aerial hyphae. However, appressorium-like structures were not observed on aerial hyphae of $F$. graminearum transformants expressing MoRAS2 ${ }^{\mathrm{G} 18 \mathrm{~V}}$. Although the formation of infection hyphae or infection cushions on plant surfaces have been reported in $F$. graminearum (Boenisch and Schafer 2011; Wanjiru et al. 2002), we failed to observe any changes in hyphal tip growth or morphology in aerial hyphae of the MoRAS2 $2^{\mathrm{G} 18 \mathrm{~V}}$ transformants of the wild type strain PH-1. These results indicated that Ras proteins in appressorium-forming fungi may have a conserved role in both surface attachment and surface recognition for regulating appressorium formation by coordinated regulation of cAMP signaling and Pmk1 MAPK pathways. Therefore, it will be important to further characterize Ras signaling in $M$. oryzae and the relationship between Ras proteins and the RA domaincontaining proteins Mac1, Mst50, and Mst11, which are the key components of these two pathways.

\section{MATERIALS AND METHODS}

\section{Strains and culture conditions.}

The wild-type and mutant strains of $M$. oryzae (Table 1) were cultured on OTA plates at $25^{\circ} \mathrm{C}$ under fluorescent light, as described (Xu and Hamer 1996). For fungal transformation, hygromycin (Calbiochem, La Jolla, CA, U.S.A.) and zeocin (Invitrogen, Carlsbad CA, U.S.A.) were added to the final concentrations of 250 and $300 \mu \mathrm{g} / \mathrm{ml}$, respectively. Monoconidial culture isolation, measurement of growth rate, and conidiation were performed as previously described (Li et al. 2004; Park et al. 2006). Vegetative hyphae from 3 -day-old $5 \times$ YEG $(0.5 \%$ yeast, $1 \%$ glucose) cultures were used for DNA and RNA isolation. For SEM examination, blocks of OTA cultures were frozen with liquid nitrogen and examined at $-140^{\circ} \mathrm{C}$ in a JEOL JSM-840 scanning electron microscope (Kong et al. 2012). For TEM examination, samples were treated and examined with a Philips/FEI CM-100 transmission electron microscope (FEI Company, Hillsboro, OR, U.S.A.), as described (Mares et al. 2006).

\section{Targeted deletion of the Ras genes in M. oryzae.}

To delete the MoRAS1 gene, the upstream and downstream flanking sequences were amplified with primer pairs RAS11F/RAS1-2R and RAS1-3F/RAS1-4R, respectively (Supplementary Table S1). The resulting PCR products were digested and ligated with the hygromycin resistance cassette $(h p h)$ released from pCX63m as described (Zhao et al. 2004). The gene-replacement construct was amplified with primers RAS1$1 \mathrm{~F}$ and RAS1-4R and directly transformed into protoplasts of 70-15. Hygromycin-resistant transformants were screened by PCR and confirmed by Southern blot analysis. A similar strategy was used to generate gene-replacement constructs for the MoRAS2 gene.

\section{Generation of the MoRAS2 ${ }^{\mathrm{G} 18 \mathrm{~V}}$ allele and transformants.}

The yeast gap repair method (Bruno et al. 2004) was used to generate the dominant active allele of MoRAS2. PCR products amplified with primer pairs RDAF1/RDAR1 and RDAF2/ RDAR2 were co-transformed into the yeast strain XK1-25 with XhoI-digested plasmid pYP1 (Zhou et al. 2011). The underlined nucleotides in the sequences of primers RDAR 1 and RDAF2 were the mutation sites. The MoRAS2 ${ }^{\mathrm{G} 18 \mathrm{~V}}$ construct named pHZ90 was isolated from the resulting Trp+ transformant and confirmed by sequencing analysis. After transformation of 70-15 with pHZ90, bleomycin-resistant transformants, including strains DA66, HZ90, HZ91, and HZ92 (Table 1), were confirmed by PCR to contain the MoRAS2 $2^{\mathrm{G} 18 \mathrm{~V}}$ construct.

Plasmid pHZ90 also was transformed into the pmkl mutant nn78 (Xu and Hamer 1996) and the cpkA mutant I27 (Mitchell and Dean 1995) to generate transformants DA-P1 and DA-C1, respectively. Transformation of $C$. graminicola, $C$. gloeosporioides, and $F$. graminearum with pHZ90 was performed as described (Armesto et al. 2012; Thon et al. 2000; Zhou et al. 2010). The resulting bleomycin-resistant transformants were confirmed by PCR to contain the MoRAS2 ${ }^{\mathrm{G} 18 \mathrm{~V}}$ construct.

\section{Generation of GFP fusion transformants,}

The GAS1-GFP fusion construct pHZ104 (Xue et al. 2002) also was transformed into protoplasts of DA66. The resulting 
zeocin-resistant transformants were assayed by PCR and GFP signals for the presence of the transforming GAS1-GFP fusion construct.

\section{Appressorium formation, penetration, and plant infection assays.}

Conidia were harvested from 10-day-old OTA cultures and resuspended to $5 \times 10^{4}$ conidia/ml. For appressorium formation assays, 50- $\mu \mathrm{l}$ droplets of conidium suspensions were placed on glass cover slips (Fisher Scientific, St. Louis) or GelBond membranes (Cambrex, East Rutherford, NJ, U.S.A.) and incubated at $25^{\circ} \mathrm{C}$ (Liu et al. 2007). Rice seedlings (2 weeks old) of 'CO-39' or 'Nipponbare' were used for spray infection assays. For infection assays with culture blocks, rice seedlings were inoculated with 1 - to $2-\mathrm{mm}^{2}$ blocks from 10 day-old OTA cultures. Lesion formation was examined 5 days postinoculation.

\section{Assays for the TEY (Thr-Glu-Tyr) phosphorylation, intracellular cAMP, and PKA activities.}

Fungal biomasses were harvested from 2-week-old OTA plates or 2-day-old liquid CM cultures and used for protein isolation or cAMP extraction (Nishimura et al. 2003; Zhao et al. 2005). Total proteins were separated on a $12.5 \%$ sodium dodecyl sulfate polyacrylamide gel electrophoresis gel and transferred to nitrocellulose membranes for Western blot analysis. TEY phosphorylation was assayed with the PhophoPlus p44/42 MAPK antibody kit following the manufacturer's protocol (Cell Signaling Technology, Danvers, MA, U.S.A.). Intracellular cAMP was assayed as described (Nishimura et al. 2003; Zhao et al. 2005) with the cAMP enzyme immunoassay system (GE Healthcare Biosciences, Pittsburg, PA, U.S.A.). PKA activities were assayed with the PepTag nonradioactive PKA assay kit (Promega Corp., Madison, WI, U.S.A.) as described (Adachi and Hamer 1998).

\section{ACKNOWLEDGMENTS}

We thank C. Woloshuk and S. Goodwin for critical reading of this manuscript and G. Park and G. Li for insightful discussions. This work was supported by a grant from the National Research Initiative of the United States Department of Agriculture NIFA (award number 201065110-20439).

\section{LITERATURE CITED}

Adachi, K., and Hamer, J. E. 1998. Divergent cAMP signaling pathways regulate growth and pathogenesis in the rice blast fungus Magnaporthe grisea. Plant Cell 10:1361-1373.

Armesto, C., Maia, F. G. M., de Abreu, M. S., Figueira, A. D., da Silva, B. M., and Monteiro, F. P. 2012. Genetic Transformation with the GFP gene of Colletotrichum Gloeosporioides isolates from coffee with blister spot. Braz. J. Microbiol. 43:1222-1229.

Belden, W. J., Larrondo, L. F., Froehlich, A. C., Shi, M., Chen, C. H., Loros, J. J., and Dunlap, J. C. 2007. The band mutation in Neurospora crassa is a dominant allele of ras-1 implicating RAS signaling in circadian output. Gene Dev. 21:1494-1505.

Bluhm, B. H., Zhao, X., Flaherty, J. E., Xu, J. R., and Dunkle, L. D. 2007. Ras2 regulates growth and pathogenesis in Fusarium graminearum. Mol. Plant-Microbe Interact. 20:627-636.

Boenisch, M. J., and Schafer, W. 2011. Fusarium graminearum forms mycotoxin producing infection structures on wheat. BMC Plant Biol. 11:110.

Boyce, K. J., Hynes, M. J., and Andrianopoulos, A. 2005. The Ras and Rho GTPases genetically interact to co-ordinately regulate cell polarity during development in Penicillium marneffei. Mol. Microbiol. 55:14871501.

Bruno, K. S., Tenjo, F., Li, L., Hamer, J. E., and Xu, J. R. 2004. Cellular localization and role of kinase activity of PMK1 in Magnaporthe grisea. Eukaryot. Cell 3:1525-1532.

Chao, C. C. T., and Ellingboe, A. H. 1991. Selection for mating compe- tence in Magnaporthe grisea pathogenic to rice. Can. J. Bot. 69: 21302134.

Chen, C. B., Ha, Y. S., Min, J. Y., Memmott, S. D., and Dickman, M. B. 2006. Cdc42 is required for proper growth and development in the fungal pathogen Colletotrichum trifolii. Eukaryot. Cell 5:155-166.

Choi, W. B., and Dean, R. A. 1997. The adenylate cyclase gene MAC1 of Magnaporthe grisea controls appressorium formation and other aspects of growth and development. Plant Cell 9:1973-1983.

Ebbole, D. J. 2007. Magnaporthe as a model for understanding hostpathogen interactions. Annu. Rev. Phytopathol. 45:437-456.

Fang, E. G. C., and Dean, R. A. 2000. Site-directed mutagenesis of the $m a g B$ gene affects growth and development in Magnaporthe grisea. Mol. Plant-Microbe Interact. 13:1214-1227.

Fernandes, L., Paes, H. C., Tavares, A. H., Silva, S. S., Dantas, A., Soares, C. M. A., Torres, F. A. G., and Felipe, M. S. S. 2008. Transcriptional profile of ras 1 and $\operatorname{ras} 2$ and the potential role of farnesylation in the dimorphism of the human pathogen Paracoccidioides brasiliensis. FEMS (Fed. Eur. Microbiol. Soc.) Yeast Res. 8:300-310.

Fillinger, S., Chaveroche, M. K., Shimizu, K., Keller, N., and d'Enfert, C. 2002. cAMP and ras signalling independently control spore germination in the filamentous fungus Aspergillus nidulans. Mol. Microbiol. 44:1001-1016.

Fortwendel, J. R., Panepinto, J. C., Seitz, A. E., Askew, D. S., and Rhodes, J. C. 2004. Aspergillus fumigatus ras $A$ and $\operatorname{ras} B$ regulate the timing and morphology of asexual development. Fungal Genet. Biol. 41:129-139.

Hlavata, L., Aguilaniu, H., Pichova, A., and Nystrom, T. 2003. The oncogenic RAS2(val19) mutation locks respiration, independently of PKA, in a mode prone to generate ROS. EMBO (Eur. Mol. Biol. Organ.) J. 22:4577A-4577A.

Kong, L. A., Yang, J., Li, G. T., Qi, L. L., Zhang, Y. J., Wang, C. F., Zhao, W. S., Xu, J. R., and Peng, Y. L. 2012. Different chitin synthase genes are required for various developmental and plant infection processes in the rice blast fungus Magnaporthe oryzae. PLoS Pathog. 8:e1002526. Published online.

Lee, N., and Kronstad, J. W. 2002. ras 2 controls morphogenesis, pheromone response, and pathogenicity in the fungal pathogen Ustilago maydis. Eukaryot. Cell 1:954-966.

Lee, N., D’Souza, C. A., and Kronstad, J. W. 2003. Of smuts, blasts, mildews, and blights: cAMP signaling in phytopathogenic fungi. Annu. Rev. Phytopathol. 41:399-427.

Lengeler, K. B., Davidson, R. C., D’Souza, C., Harashima, T., Shen, W. C., Wang, P., Pan, X. W., Waugh, M., and Heitman, J. 2000. Signal transduction cascades regulating fungal development and virulence. Microbiol. Mol. Biol. Rev. 64:746-785.

Leung, H., Borromeo, E. S., Bernardo, M. A., and Notteghem, J. L. 1988 Genetic analysis of virulence in the rice blast fungus Magnaporthe grisea. Phytopathology 78: 1227-1233.

Li, G., Zhou, X., and Xu, J.-R. 2012. Genetic control of infection-related development in Magnaporthe oryzae. Curr. Opin. Microbiol. 15:678684.

Li, L., Xue, C. Y., Bruno, K., Nishimura, M., and Xu, J. R. 2004. Two PAK kinase genes, CHM1 and MST20, have distinct functions in Magnaporthe grisea. Mol. Plant-Microbe Interact. 17:547-556.

Liu, H., Suresh, A., Willard, F. S., Siderovski, D. P., Lu, S., and Naqvi, N. I. 2007. Rgs1 regulates multiple $\mathrm{G}$ alpha subunits in Magnaporthe pathogenesis, asexual growth and thigmotropism. EMBO (Eur. Mol. Biol. Organ.) J. 26:690-700.

Lorenz, M. C., and Heitman, J. 1997. Yeast pseudohyphal growth is regulated by GPA2, a G protein alpha homolog. EMBO (Eur. Mol. Biol. Organ.) J. 16:7008-7018.

Mares, D., Romagnoli, C., Andreotti, E., Forlani, G., Guccione, S., and Vicentini, C. B. 2006. Emerging antifungal azoles and effects on Magnaporthe grisea. Mycol. Res. 110:686-696.

McDonough, K. A., and Rodriguez, A. 2012. The myriad roles of cyclic AMP in microbial pathogens: From signal to sword. Nat. Rev. Microbiol. 10:27-38.

Mitchell, T. K., and Dean, R. A. 1995. The cAMP-dependent protein kinase catalytic subunit is required for appressorium formation and pathogenesis by the rice blast pathogen Magnaporthe grisea. Plant Cell 7:18691878 .

Mosch, H. U., Roberts, R. L., and Fink, G. R. 1996. Ras2 signals via the $\mathrm{Cdc} 42 / \mathrm{Ste} 20 / \mathrm{mitogen}$-activated protein kinase module to induce filamentous growth in Saccharomyces cerevisiae. Proc. Natl. Acad. Sci. U.S.A. 93:5352-5356.

Mosch, H. U., Kubler, E., Krappmann, S., Fink, G. R., and Braus, G. H. 1999. Crosstalk between the Ras2p-controlled mitogen-activated protein kinase and cAMP pathways during invasive growth of Saccharomyces cerevisiae. Mol. Biol. Cell 10:1325-1335.

Muller, P., Katzenberger, J. D., Loubradou, G., and Kahmann, R. 2003. Guanyl nucleotide exchange factor Sq12 and Ras2 regulate filamentous 
growth in Ustilago maydis. Eukaryot. Cell 2:609-617.

Nichols, C. B., Ferreyra, J., Ballou, E. R., and Alspaugh, J. A. 2009. Subcellular localization directs signaling specificity of the Cryptococcus neoformans Ras1 protein. Eukaryot. Cell 8:181-189.

Nishimura, M., Park, G., and Xu, J. R. 2003. The G-beta subunit MGB1 is involved in regulating multiple steps of infection-related morphogenesis in Magnaporthe grisea. Mol. Microbiol. 50:231-243.

Onken, B., Wiener, H., Philips, M. R., and Chang, E. C. 2006. Compartmentalized signaling of Ras in fission yeast. Proc. Natl. Acad. Sci. U.S.A. 103:9045-9050.

Park, G., Xue, C., Zhao, X., Kim, Y., Orbach, M., and Xu, J. R. 2006. Multiple upstream signals converge on the adaptor protein Mst50 in Magnaporthe grisea. Plant Cell 18:2822-2835.

Saunders, D. G. O., Dagdas, Y. F., and Talbot, N. J. 2010. Spatial uncoupling of mitosis and cytokinesis during appressorium-mediated plant infection by the rice blast fungus Magnaporthe oryzae. Plant Cell 22:2417-2428.

Schubert, D., Raudaskoski, M., Knabe, N., and Kothe, E. 2006. Ras GTPase-activating protein Gap1 of the homobasidiomycete Schizophyllum commune regulates hyphal growth orientation and sexual development. Eukaryot. Cell 5:683-695.

Shapiro, R. S., Robbins, N., and Cowen, L. E. 2011. Regulatory circuitry governing fungal development, drug resistance, and disease. Microbiol. Mol. Biol. Rev. 75:213-267.

Soanes, D. M., Chakrabarti, A., Paszkiewicz, K. H., Dawe, A. L., and Talbot, N. J. 2012. Genome-wide transcriptional profiling of appressorium development by the rice blast fungus Magnaporthe oryzae. PLoS Pathog. 8:e1002514. Published online.

Tamanoi, F. 2011. Ras signaling in yeast. Genes Cancer 2:210-215.

Thon, M. R., Nuckles, E. M., and Vaillancourt, L. J. 2000. Restriction enzyme-mediated integration used to produce pathogenicity mutants of Colletotrichum graminicola. Mol. Plant-Microbe Interact. 13:13561365.

Villalba, F., Collemare, J., Landraud, P., Lambou, K., Brozek, V., Cirer, B., Morin, D., Bruel, C., Beffa, R., and Lebrun, M. H. 2008. Improved gene targeting in Magnaporthe grisea by inactivation of MgKU80 required for non-homologous end joining. Fungal Genet. Biol. 45: 68-75.

Wanjiru, W. M., Kang, Z. S., and Buchenauer, H. 2002. Importance of cell wall degrading enzymes produced by Fusarium graminearum during infection of wheat heads. Eur. J. Plant Pathol. 108:803-810.

Waugh, M. S., Nichols, C. B., DeCesare, C. M., Cox, G. M., Heitman, J., and Alspaugh, J. A. 2002. Ras1 and Ras2 contribute shared and unique roles in physiology and virulence of Cryptococcus neoformans. Microbiology 148:191-201

Wilson, R. A., and Talbot, N. J. 2009. Under pressure: Investigating the biology of plant infection by Magnaporthe oryzae. Nat. Rev. Microbiol. 7:185-195.

Xie, X. Q., Guan, Y., Ying, S. H., and Feng, M. G. 2013. Differentiated functions of Ras1 and Ras2 proteins in regulating the germination, growth, conidiation, multi-stress tolerance and virulence of Beauveria bassiana. Environ. Microbiol. 15:447-462.

Xu, J. R., and Hamer, J. E. 1996. MAP kinase and cAMP signaling regulate infection structure formation and pathogenic growth in the rice blast fungus Magnaporthe grisea. Gene Dev. 10:2696-2706.

Xue, C. Y., Park, G., Choi, W. B., Zheng, L., Dean, R. A., and Xu, J. R. 2002. Two novel fungal virulence genes specifically expressed in appressoria of the rice blast fungus. Plant Cell 14:2107-2119.

Zhao, X. H., and Xu, J. R. 2007. A highly conserved MAPK-docking site in Mst7 is essential for Pmk1 activation in Magnaporthe grisea. Mol. Microbiol. 63:881-894.

Zhao, X. H., Xue, C., Kim, Y., and Xu, J. R. 2004. A ligation-PCR approach for generating gene replacement constructs in Magnaporthe grisea. Fungal Genet. Newsl. 51:17-18.

Zhao, X. H., Kim, Y., Park, G., and Xu, J. R. 2005. A mitogen-activated protein kinase cascade regulating infection-related morphogenesis in Magnaporthe grisea. Plant Cell 17:1317-1329.

Zhou, X. Y., Heyer, C., Choi, Y. E., Mehrabi, R., and Xu, J. R. 2010. The CID1 cyclin C-like gene is important for plant infection in Fusarium graminearum. Fungal Genet. Biol. 47:143-151.

Zhou, X. Y., Li, G. T., and Xu, J. R. 2011. Efficient approaches for generating GFP fusion and epitope-tagging constructs in filamentous fungi. Pages 199-212 in: Fungal Genomics. J. R. Xu and H. B. Bluhm, eds. Humana Press, New York.

Zhou, X. Y., Zhang, H. F., Li, G. T., Shaw, B., and Xu, J. R. 2012. The cyclase-associated protein Cap1 is important for proper regulation of infection-related morphogenesis in Magnaporthe oryzae. PLoS Pathog. 8:e100291. doi:100210.101371/journal.ppat.1002911. Published online.

Zhu, Y., Fang, H. M., Wang, Y. M., Zeng, G. S., Zheng, X. D., and Wang, Y. 2009. Ras1 and Ras 2 play antagonistic roles in regulating cellular cAMP level, stationary-phase entry and stress response in Candida albicans. Mol. Microbiol. 74:862-875.

Zisoulis, D. G., and Kansas, G. S. 2004. H-Ras and phosphoinositide 3kinase cooperate to induce alpha(1,3)-fucosyltransferase VII expression in Jurkat T cells. J. Biol. Chem. 279:39495-39504. 